\title{
Cirurgia de catarata: características e opiniões de pacientes com visão mono versus binocular
}

\author{
Cataract surgery: characteristics and opinions of patients with monocular \\ versus binocular vision
}

Roberta Ferrari Marback ${ }^{1}$, Edméa Rita TemporinI², Newton Kara Júnior ${ }^{3}$

\section{RESUMO}

Objetivo: Verificar em dois grupos de pacientes com visão monocular (grupo 1 ) e com visão binocular (grupo 2), a serem submetidos à cirurgia de catarata num hospital universitário, opiniões em relação ao problema ocular, à qualidade da visão e à cirurgia de catarata.

Métodos: Foi realizado estudo transversal e comparativo, de forma consecutiva, por meio de questionário estruturado, aplicado por entrevista a pacientes, elaborado a partir de estudo exploratório e medidas acuidade visual e causa da perda visual.

Resultados: A amostra foi constituída por 96 indivíduos do grupo 1 (50,0\% homens; 50,0\% mulheres, com idade entre 41 e 91 anos, média 69,3 anos 10,4 anos) e 110 do grupo 2 (40,9\% homens. $59,1 \%$ mulheres, com idade entre 40 e 89 anos, média 68,2 anos $\pm 10,2$ anos). A maioria dos indivíduos de ambos os grupos apresentava baixa escolaridade. Não houve diferença estatisticamente significante entre os grupos em relação ao sexo $(p=0,191)$, à idade $(p=0,702)$ e à escolaridade $(p=0,245)$. Não exerciam atividade laboral $95,8 \%$ dos indivíduos do grupo 1 e 83,6\%, do grupo 2 ( $p=0,005)$ e 30,4\% do grupo 1 mencionaram não ter possibilidade de trabalhar por causa da deficiência visual. Observou-se acuidade visual do olho a ser operado menor que 0,05 em $40,6 \%$ (grupo 1) e 33,6\% (grupo 2), entre 0,25 e 0,05. Quase a totalidade dos indivíduos de ambos os grupos afirmou ter dificuldade para realização das atividades de vida diária e qualificou como insuficiente a respectiva acuidade visual: $71,9 \%$ dos entrevistados do grupo 1 e $71,6 \%$, do grupo 2 mencionaram saber a causa da visão fraca; desses, $87,1 \%$ do grupo 1 e $83,3 \%$ do grupo 2 referiram a catarata como causa da baixa acuidade visual.

Conclusão: Os indivíduos de ambos os grupos tiveram acesso à cirurgia de catarata com acuidade visual menor do que a idealmente indicada; os pacientes com visão monocular apresentaram acuidade visual significativamente meno em relação aos com visão binocular; a maioria dos entrevistados de ambos os grupos referiu dificuldades para realizar atividades cotidianas como consequência da baixa visão; muitos indivíduos de ambos os grupos desconheciam a causa da dificuldade visual ou a atribuíram a outra causa que não a catarata.

Descritores: Extração de catarata; Visão monocular; Visão binocular; Acuidade visual; Atividades cotidianas; Qualidade de vida; Questionários

\section{ABSTRACT}

Purpose: To verify in two groups of patients: monocular (group 1) and binocular vision (group 2) to be submitted to cataract surgery at an University Hospital, opinions, expectances and emotional reactions related to the ocular problem, to the quality of vision and to cataract surgery.

Methods: A transversal comparative and consecutive study was performed using a structured questionnaire applied by patients interview. The questionnaire was elaborated from a previous exploratory study; visual acuity and cause of the visual loss were evaluated.

Results: The sample was constituted by 96 persons of group 1 (50.0\% male; $50.0 \%$ female, ages ranging from 41 to 91 years; average 69.3 years \pm 10.4 years) and 110 persons of group $2(40.9 \%$ male; $59.1 \%$ female, ages ranging from 40 to 89 years; average 68.2 years \pm 10.2 years). The majority of persons of both groups presented low educational level. There was no statistically significant difference between the groups in relation to gender $(p=0.191)$, age $(p=0.702)$ and educational level ( $p=0.245$ ). No workactivity was mentioned in $95.8 \%$ of the persons of group 1 and $83.6 \%$ of group $2(p=0.005)$ and $30.4 \%$ of group 1 informed the impossibility to work due the visual impairment Visual acuity of the eye to be operated was less than 0.05 in $40.6 \%$ (group 1) and in 33,6\% (group 2), presented visual acuity ranging from 0.05 to 0.25 . Almost the totality of the persons of both groups informed difficulties to perform activities of daily life and qualified as insufficient their visual acuities: $71.9 \%$ of the patients of group 1 and $71.6 \%$ of group 2 informed to know the reason of low vision; among these, $87.1 \%$ of group 1 and $83.3 \%$ of group 2 mentioned cataract as the reason of low visual acuity. Conclusion: It was concluded that the patients of both groups were submitted to cataract surgery with visual acuities less than the visual acuity ideally indicated; the patients with monocular vision showed visual acuities significantly less in relation to the patients with binocular vision; the majority of the patients of both groups mentioned difficulties to perform daily activities as a consequence of low vision; patients with monocular vision mentioned doubts in relation to the surgical results as compared with the patients with binocular vision; many patients of both groups did not know the cause of the visual difficulty or explained the visual difficulty by other cause than the cataract.

Keywords: Cataract extraction; Vision, monocular; Vision, binocular; Visual acuity; Activities of daily living; Quality of life; Questionnaires
Psicóloga, Hospital das Clínicas, Faculdade de Medicina, Universidade de São Paulo - USP - São Paulo (SP), Brasil.

Sanitarista, Hospital das Clínicas, Faculdade de Medicina, Universidade de São Paulo - USP - São Paulo (SP), Brasil.

3 Médico, Hospital das Clínicas, Faculdade de Medicina. Universidade de São Paulo - USP São Paulo (SP), Brasil.

Endereço para correspondência: Roberta Ferrari Marback. Rua Eduardo José dos Santos, 147 - Centro Integrado de Saúde Professor Fernando Filgueiras - Salas $807 / 808$

147 - Centro Integrado de Saúde Professor Fernando Filgueiras - Salas 807/8
Salvador (BA) - CEP 41940-455 - E-mail: robertamarback@uol.com.br

Recebido para publicação em 02.12.2009

Aprovado em 31.07.2010

Baseado na Tese de Doutorado Intitulada "Cirurgia de catarata: opiniões, expectativas e reações emocionais de pacientes com visão mono versus binocular", defendida no Departamento de Oftalmologia da Faculdade de Medicina da Universidade de São Paulo, em tamento de Oftalmologia da Faculdade de Medicina da Universidade de São Paulo, em Nível Superior (CAPES)

Nota Editorial: Depois de concluída a análise do artigo sob sigilo editorial e com a anuência da Dra. Amélia Kamegasawa sobre a divulgação de seu nome como revisora, agradecemos sua participação neste processo.

\section{INTRODUÇÃO}

1 cegueira e a deficiência visual são consideradas um grave problema de saúde pública. Para a Organização Mundial de Saúde (OMS), uma pessoa é considerada cega quando apresenta acuidade visual (AV) menor do que 0,05 no melhor olho (pela tabela de Snellen), assim como a incapacidade visual (baixa visão) é definida como acuidade visual menor do que 0,10 no melhor olho, com a melhor correção óptica(1). Nos casos de haver perda visual grave somente em um dos olhos, a pessoa não é considerada cega, mas com um olho cego.

A incidência anual de deficiência visual por catarata senil é de 2 a 3 casos para cada 1.000 habitantes, segundo dados da $\mathrm{OMS}^{(2)}$. A catarata é responsável por cerca de $50 \%$ dos casos de cegueira no Brasil(3) 
Ações para diminuição da cegueira por catarata, no Brasil, têm sido realizadas desde 1986, quando tiveram início as atividades do Projeto Catarata $^{(3)}$. Sabe-se que, para um indivíduo se submeter à cirurgia de catarata, além de preencher os prérequisitos clínicos para a indicação cirúrgica e de ter condições de acesso à assistência especializada, também é necessário estar ciente do seu problema ocular, ter conhecimento da existência de tratamento, obter confiança na possibilidade de recuperação visual e aceitar o tratamento cirúrgico proposto ${ }^{(4-6)}$.

Acredita-se que, algumas das barreiras, para que os indivíduos se submetam à cirurgia de catarata, têm sido geradas por fatores psicológicos e culturais dos pacientes, relacionados à importância da visão para a qualidade de vida ${ }^{(4-5)}$. Assim, é possível que pessoas, que por algum motivo, não possuam potencial visual em um dos olhos (visão monocular), e necessitem ser submetidas à cirurgia de catarata no único olho funcional, apresentem opiniões diferentes sobre o problema ocular e seu tratamento, daquelas com potencial visual em ambos os olhos (visão binocular), o que pode influenciar na aceitação do tratamento cirúrgico.

O presente estudo teve como escopo comparar, em dois grupos, de pacientes com visão monocular (grupo 1) e com visão binocular (grupo 2), a serem submetidos à cirurgia de catarata num hospital universitário, características visuais e opiniões em relação ao problema ocular, à qualidade da visão e à cirurgia de catarata.

\section{MÉTODOS}

Foi realizado estudo transversal e comparativo. A amostra foi composta por 206 indivíduos de ambos os sexos, a serem submetidos à cirurgia de catarata, com idade superior a 40 anos, atendidos de forma consecutiva pela Clínica Oftalmológica de um hospital universitário, que se dispuseram a ser entrevistados. Foram formados dois grupos para efeitos de comparação, por amostragem não-probabilística: o grupo 1, constituído por indivíduos com visão monocular, a serem submetidos à cirurgia de catarata no único olho funcional; e, o grupo 2, composto por indivíduos com visão binocular, a serem submetidos à cirurgia de catarata, pela primeira vez, no olho de pior visão.

Considerou-se que o indivíduo apresentava visão monocular se diagnosticada cegueira legal irreversível em um dos olhos - acuidade visual menor ou igual a 0,10 (20/200) pela tabela de Snellen ou campo visual menor do que 20 graus ${ }^{(7)}$; e visão binocular se, apesar da opacidade do cristalino, ambos os olhos apresentavam potencial visual, com possibilidade de melhora significativa da acuidade visual, após a cirurgia de catarata.

Diante da diversidade das características socioculturais, optou-se por realizar o estudo por meio de questionário elaborado a partir de pesquisa exploratória, entre pacientes com características semelhantes às da amostra ${ }^{(5-6,8-9)}$. A coleta de dados foi realizada no período de março de 2006 a março de 2007, no Centro Cirúrgico Ambulatorial do hospital universitário, no momento em que os entrevistados aguardavam a cirurgia. Considerando a natureza de cada grupo, o questionário, para o grupo 2, foi acrescido de duas questões ( 6 e 7), tendo sido as outras comuns para ambos os grupos (Anexo).

Antes de iniciar as entrevistas, foram explicitados os objetivos e método da pesquisa, bem como assegurados sigilo e anonimato das informações por eles fornecidas, além da ausência de agravos ou prejuízos em relação ao tratamento oferecido na clínica oftal- mológica. Os sujeitos assinaram termo de consentimento livre e esclarecido. Este estudo foi aprovado pela Comissão de Ética da instituição.

\section{RESULTADOS}

O grupo 1 foi composto por 96 sujeitos, $50,0 \%$ do sexo feminino e 50,0\%, masculino. A idade variou de 41 a 91 anos, com média de 69,3 anos \pm 10,4 anos; 29,2\% tinham entre 60 e 69 anos e 36,4\%, entre 70 e 79 anos. Quanto à escolaridade, $22,9 \%$ não estudaram e $61,5 \%$ cursaram o ensino fundamental incompleto. O grupo 2 foi constituído por 110 sujeitos, 59,1\% do sexo feminino e 40,9\%, masculino. A idade variou de 40 a 89 anos, com média de 68,2 anos $\pm 10,2$ anos; $29,1 \%$ tinham entre 60 e 69 anos e 42,7\%, entre 70 e 79 anos. No que tange à escolaridade, $14,5 \%$ declararam não ter estudado e 67,3\%, ter cursado o ensino fundamental incompleto. Não houve dife-
Anexo

QUESTIONÁRIO

1. Sexo:

Masculino ........................................................................... (1)

Feminino ...........................................................................................

2. Idade ... (anos)

3. $O(A)$ sr(a). estudou na escola, sim ou não? ECA:

Ensino fundamental:

Até

Até (série) s

Ensino superior:

Incompleto

Completo

Não estudou .

4. Atualmente o(a) sr(a). trabalha e ganha pelo que faz, sim ou não? ECA: No que o(a) sr(a). trabalha?

Não trabalha (1)

Trabalha (passe para a questão 6) ………………………....... (Ocupação)

5. $\mathrm{O}(\mathrm{A}) \operatorname{sr}(\mathrm{a})$. não está trabalhando porque:

Aposentou-se?

É pensionista?

Não consegue trabalho?

Não tem possibilidade de trabalhar?

(4)

Atividades do lar? .............................................................. (5)

6. Quanto tempo faz que o(a) sr(a). perdeu a visão do olho que não vai ser operado? (Tempo)

7. Na sua opinião, o que causou a perda dessa vista? Foi por: Doença na retina

Doença no nervo óptico ..................................................... (2) (1)

Doença na córnea .......................................................................

Catarata

Trauma ..............................................................................

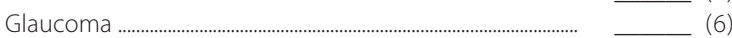

Problema de nascença .................................................... _ _ (7)

Idade ....................................................................................

Não sabe

Outro .......................................................................................

Qual?

8. Na sua opinião, a vista com a qual o(a) sr(a). enxerga está fraca? Sim ou não?

Sim ....................... (1)

Não …………………………………...... (Passe para a questão 13) $\longleftarrow$ (2)

9. O(A) sr(a). sabe a causa da visão fraca do olho que vai ser operado? Sim ou não?

Sim

Qual?

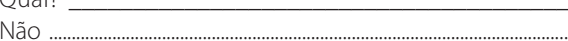


Tabela 1. Características pessoais e situação ocupacional de pacientes com visão monocular (grupo 1) e com visão binocular (grupo 2), a serem submetidos à cirurgia de catarata. HC-FMUSP - 2007

\begin{tabular}{|c|c|c|c|c|c|}
\hline \multirow[b]{2}{*}{ Características } & \multicolumn{2}{|c|}{ Grupo 1 n=96 } & \multicolumn{2}{|c|}{ Grupo $2 \mathrm{n}=110$} & \multirow[b]{2}{*}{ Valor de $p$} \\
\hline & $\mathbf{n}$ & $\%$ & $\mathbf{n}$ & $\%$ & \\
\hline \multicolumn{6}{|l|}{ Sexo } \\
\hline Masculino & 48 & 50,0 & 45 & 40,9 & \\
\hline Feminino & 48 & 50,0 & 65 & 59,1 & 0,191 \\
\hline & \multicolumn{2}{|c|}{$n=39$} & \multicolumn{2}{|c|}{$n=25$} & \\
\hline \multicolumn{6}{|l|}{ Idade (anos) } \\
\hline $40 \vdash 60$ & 17 & 17,7 & 18 & 16,4 & \\
\hline $60 \vdash 70$ & 28 & 29,2 & 32 & 29,1 & \\
\hline $70 \vdash 80$ & 35 & 36,4 & 47 & 42,7 & \\
\hline $80 \vdash 91$ & 16 & 16,7 & 13 & 11,8 & 0,702 \\
\hline$\overline{\mathrm{x}} \pm \mathrm{DP}$ & \multicolumn{2}{|c|}{$69,3 \pm 10,4$ anos } & \multicolumn{2}{|c|}{$68,2 \pm 10,2$ anos } & \\
\hline \multicolumn{6}{|l|}{ Escolaridade } \\
\hline Não estudou & 22 & 22,9 & 16 & 14,5 & \\
\hline \multicolumn{6}{|l|}{ Ensino fundamental } \\
\hline Incompleto & 59 & 61,5 & 74 & 67,3 & \\
\hline Completo & 2 & 2,1 & 8 & 7,3 & \\
\hline \multicolumn{6}{|l|}{ Ensino médio } \\
\hline Incompleto & 1 & 1,0 & 2 & 2,8 & \\
\hline Completo & 6 & 6,2 & 7 & 6,4 & \\
\hline \multicolumn{6}{|l|}{ Ensino superior } \\
\hline Incompleto & 2 & 2,1 & - & - & \\
\hline Completo & 4 & 4,2 & 3 & 2,7 & 0,245 \\
\hline \multicolumn{6}{|l|}{ Situação ocupacional } \\
\hline Trabalha com remuneração & 4 & 4,2 & 18 & 16,4 & \\
\hline Não trabalha & 92 & 95,8 & 92 & 83,6 & $0,005^{*}$ \\
\hline Razões & \multicolumn{2}{|c|}{$\mathrm{n}=92$} & \multicolumn{2}{|c|}{$\mathrm{n}=92$} & \\
\hline Aposentou-se pela idade & 47 & 51,1 & 62 & 67,4 & \\
\hline Não tem possibilidade de trabalhar por & & & & & \\
\hline causa da deficiência visual & 28 & 30,4 & 4 & 4,3 & \\
\hline Atividades do lar & 9 & 9,8 & 10 & 10,9 & \\
\hline Recebe pensão & 8 & 8,7 & 15 & 16,3 & \\
\hline Não consegue trabalho & - & - & 1 & 1,1 & $0,001^{*}$ \\
\hline
\end{tabular}

* =t este qui-quadrado; $\overline{\mathrm{x}}=$ média; $\mathrm{DP}=$ desvio padrão

Tabela 2. Acuidade visual do olho a ser operado e do olho contralateral. Pacientes com visão monocular (grupo 1) e visão binocular (grupo 2), a serem submetidos à cirurgia de catarata. HC-FMUSP - 2007

\begin{tabular}{|c|c|c|c|c|c|}
\hline \multirow[b]{2}{*}{ Acuidade visual } & \multicolumn{2}{|c|}{ Grupo $1 \mathrm{n}=96$} & \multicolumn{2}{|c|}{ Grupo $2 n=110$} & \multirow{2}{*}{$\begin{array}{l}\text { Valor } \\
\text { de } p^{*}\end{array}$} \\
\hline & $n$ & $\%$ & $n$ & $\%$ & \\
\hline \multicolumn{6}{|l|}{ Olho a ser operado } \\
\hline$>0,50$ & - & - & 2 & 1,8 & \\
\hline $0,50 \vdash 0,30$ & 8 & 8,3 & 27 & 24,6 & \\
\hline $0,30 \vdash 0,25^{c}$ & 16 & 16,7 & 23 & 20,9 & \\
\hline $0,25 \vdash 0,10^{d}$ & 22 & 22,9 & 26 & 23,6 & \\
\hline $0,10 \vdash 0,05^{\mathrm{e}}$ & 11 & 11,5 & 11 & 10,0 & \\
\hline$<0,05^{f}$ & 39 & 40,6 & 21 & 19,1 & 0,002 \\
\hline \multicolumn{6}{|l|}{ Olho contralateral ${ }^{b}$} \\
\hline$>0,50$ & - & - & 24 & 21,8 & \\
\hline $0,50 \vdash 0,30$ & - & - & 41 & 37,3 & \\
\hline $0,30 \vdash 0,25^{c}$ & - & - & 22 & 20,0 & \\
\hline $0,25 \vdash 0,10^{d}$ & - & - & 18 & 16,4 & \\
\hline $0,10 \vdash 0,05^{e}$ & 5 & 5,2 & 1 & 0,9 & \\
\hline$<0,05^{f}$ & 91 & 94,8 & 4 & 3,6 & $<0,001$ \\
\hline
\end{tabular}

${ }^{a}=$ no grupo 1 , refere-se à visão monocular; ${ }^{b}=$ no grupo 1, refere-se ao ol ho cego; ${ }^{c}=$ baixa visão leve; ${ }^{d}=$ baixa visão moderada; ${ }^{e}=$ baixa visão grave; ${ }^{f}=$ baixa visão profunda $(\mathrm{OMS}, 2001){ }^{*}=$ teste qui-quadrado

rença, estatisticamente significante, entre os grupos, em relação ao sexo $(p=0,191)$, à idade $(p=0,702)$ e à escolaridade $(p=0,245)$ (Tabela 1).
Em relação à situação ocupacional, 95,8\% do grupo 1 não exerciam atividade remunerada; desses, 51,1\% eram aposentados e 30,4\% referiram não ter possibilidade de trabalhar em razão da deficiência visual. No grupo 2, 83,6\% não exerciam atividade remunerada; desses, 67,4\% eram aposentados. Houve diferença, estatisticamente significante, entre os grupos, em relação à situação ocupacional $(p=0,005)$ e as razões por não trabalhar $(p=0,001)$ (Tabela 1).

No grupo 1, a medida da visão do olho funcional revelou $40,6 \%$ de sujeitos que apresentavam AV igual ou menor do que 0,$05 ; 11,5 \%$ entre 0,10 e 0,$05 ; 22,9 \%$, entre 0,25 e 0,10. Em relação ao olho cego, 94,8\% tinham AV menor que 0,05 . No grupo 2,19,1\% tinham visão no olho a ser operado menor que 0,05; 10,0\%, entre 0,10 e 0,05 e 44,5\%, entre 0,30 e 0,10. Quanto ao olho contralateral, 3,6\% dos pacientes possuíam AV menor que 0,$05 ; 17,3 \%$, entre de 0,25 e 0,$05 ; 20,0 \%$ entre 0,30 e 0,25 e 59,1\% acima de 0,3. Houve diferença, estatisticamente significante na $A V$, entre os dois grupos, tanto em relação ao olho a ser operado $(p=0,002)$, quanto ao olho contralateral $(p<0,001)$ (Tabela 2).

Os pacientes do grupo $1(n=96)$ referiram o tempo e a causa da perda visual do olho não funcional: mais de 20 anos (38,5\%); entre 5 e 20 anos $(26,1 \%)$ e menos de 5 anos $(35,4 \%)$ e apontaram como causa da perda visual o glaucoma (21,9\%); trauma $(20,8 \%)$ e doença na retina $(19,8 \%)$. Quanto ao diagnóstico médico $(n=78)$, foi confirmado durante o exame pré-operatório que $43,6 \%$ apresentavam doença na retina; $19,2 \%$, sinais de 
trauma ocular e 18,0\%, glaucoma. Do total de pacientes, 18 sujeitos (18,7\%) não dispunham de prontuários ou estes se mostravam incompletos (Tabela 3).

A totalidade dos pacientes do grupo 1 referiu possuir a percepção de que a visão no olho funcional era "fraca" e 71,9\% mencionaram saber a causa da "visão fraca". Desses, 66,7\% atribuíram a causa apenas à catarata; $17,4 \%$, à catarata associada à outra doença e 15,9\%, a outro fator. No grupo 2, 99,1\% consideraram "fraca" a visão do olho a ser operado; $71,6 \%$ afirmaram saber a causa da "visão fraca". Desses, 69,2\% atribuíram a causa apenas à catarata; $14,1 \%$, à catarata associada à outra doença e $16,7 \%$, a outro fator (Tabela 4)

\section{DISCUSSÃO}

Em relação às características pessoais, observou-se que não houve diferença, estatisticamente significante, entre os grupos, quanto ao sexo $(p=0,191)$. O grupo 1 teve proporção igual entre os sexos e o grupo 2 apresentou proporção maior de mulheres $(59,1 \%)$ (Tabela 1$)$

Quanto à faixa etária, observou-se que, uma proporção significativa dos indivíduos de ambos os grupos apresentava mais de 60 anos, não havendo, no entanto, diferença estatisti- camente significante entre os grupos (Tabela 1). Estudos realizados previamente encontraram dados semelhantes ${ }^{(4,10)}$.

O nível de escolaridade foi baixo em ambos os grupos (Tabela 1). Esses dados são característicos de população carente atendida pelo serviço público em hospital universitário de país em desenvolvimento, como o Brasi|(10). Estudos com populações semelhantes, com catarata e da mesma faixa etária, realizados em países desenvolvidos, como os Estados Unidos, revelaram nível de escolaridade mais alto, 29\% dos indivíduos tinham concluído nível superior ${ }^{(11)}$. No presente estudo, a proporção mínima dos entrevistados dos grupos 1 e 2 tinham concluído o ensino superior. Acredita-se que, em populações com baixos níveis educacionais, em geral, os conceitos, a respeito da influência da catarata na acuidade visual e a possibilidade de cura por meio de cirurgia, sejam equivocados, dificultando ou retardando a procura pelo tratamento(6)

Houve diferença estatisticamente significante entre os grupos quanto à situação ocupacional, enquanto apenas 4,2\% dos indivíduos do grupo 1 referiram trabalhar remuneradamente, $16,4 \%$, do grupo 2 exerciam atividade remunerada $(p=0,005)$. Esses dados sugerem maiores dificuldades, de pessoas com catarata e visão monocular, para realização de atividades labo-

Tabela 3 . Opinião referente ao tempo decorrido e à causa da perda visual do olho cego
e diagnóstico médico. Pacientes com visão monocular, a serem submetidos à cirurgia de catarata (grupo 1). HC-FMUSP - 2007

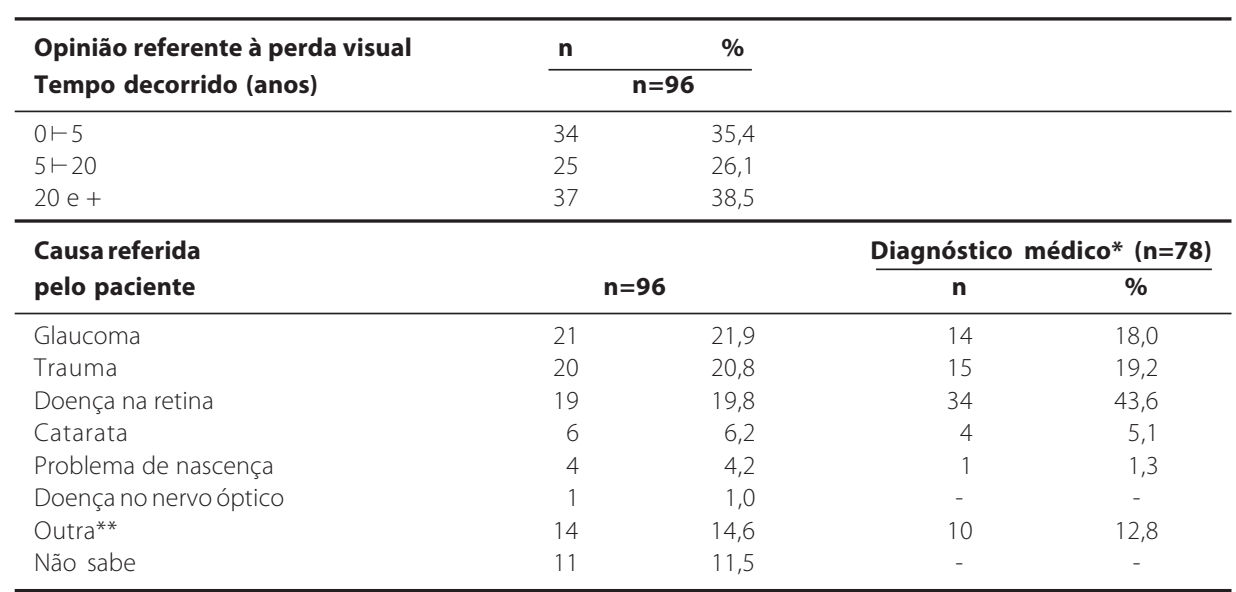

*= 18 referem-se a prontuários não localizados ou incompletos; ** = outras causas mencionadas: toxoplasmose, doença de Behçet, acidente vascular cerebral, meningioma, "o vento que passou", "o olho grudou e a visão baixou", "ritimia", "quisto no dente" e "furou o pé com prego"

Tabela 4. Opinião sobre a qualidade da visão e sobre a causa da perda visual. Pacientes com visão monocular (grupo 1) e com visão binocular (grupo 2), a serem submetidos à cirurgia de catarata. HC-FMUSP - 2007

\begin{tabular}{|c|c|c|c|c|c|}
\hline \multirow[b]{2}{*}{ Opinião } & \multicolumn{2}{|c|}{ Grupo $1 \mathrm{n}=96$} & \multicolumn{2}{|c|}{ Grupo $2 \mathrm{n}=110$} & \multirow[b]{2}{*}{ valor de $p$} \\
\hline & $\mathbf{n}$ & $\%$ & $\mathbf{n}$ & $\%$ & \\
\hline A visão está fraca & 96 & 100,0 & 109 & 99,1 & \\
\hline \multirow[t]{2}{*}{ A visão não está fraca } & - & - & 1 & 0,9 & $1,000^{*}$ \\
\hline & & \multicolumn{3}{|c|}{$n=109$} & \\
\hline Não sabe a causa da visão fraca & 27 & 28,1 & 31 & 28,4 & \\
\hline \multirow[t]{2}{*}{ Sabe a causa da visão fraca } & 69 & 71,9 & 78 & 71,6 & $0,960 * *$ \\
\hline & \multicolumn{2}{|c|}{$n=69$} & \multicolumn{2}{|c|}{$\mathrm{n}=78$} & \\
\hline Atribui a causa apenas à catarata & 46 & 66,7 & 54 & 69,2 & \\
\hline Atribui a causa à catarata associada à outra doença & 12 & 17,4 & 11 & 14,1 & \\
\hline Atribui a causa a outro fator & 11 & 15,9 & 13 & 16,7 & $0,861^{* *}$ \\
\hline
\end{tabular}

${ }^{*}=$ teste exato de Fisher; ${ }^{* *}=$ teste qui-quadrado 
rais. No grupo 1, praticamente um terço dos indivíduos que não exerciam atividade remunerada, mencionaram não ter possibilidade de trabalhar por causa da deficiência visual; enquanto que no grupo 2, apenas 4,3\% apontaram essa questão (Tabela 1). Mais da metade da amostra, em ambos os grupos, referiu ser aposentada pela idade, coerentemente à média de idade dos indivíduos ${ }^{(12)}$

O diagnóstico precoce da catarata, geralmente acompanhado do tratamento efetivo por meio da correção cirúrgica, atualmente realizado com eficácia e segurança, graças ao progresso tecnológico e ao preparo e habilidade dos cirurgiões, permite que o indivíduo não restrinja sua atividade laboral e se mantenha integrado ao processo social ${ }^{(13-14)}$

Estudos apontaram que, para maior benefício da sociedade, deve-se assegurar que os esforços no combate à catarata se concentrem, também, na prevenção da "cequeira econômica", isto é, quando a dificuldade visual passa a comprometer o desempenho profissional do indivíduo. Considera-se "cegueira econômica" quando a AV é menor que 0,30, o que limita a visão do paciente, mas ainda assim, este não tem acesso ao tratamento, ou não o procura, levando o indivíduo a abdicar de sua atividade profissional, gerando prejuízo financeiro a ele e ao país. O objetivo é evitar a exclusão do mercado de trabalho enquanto a cirurgia é aguardada, uma vez que se torna mais difícil a reintegração de uma pessoa ao mercado de trabalho após a perda do emprego(13).

Observou-se que 75,0\% dos pacientes do grupo 1 apresentaram AV no único olho funcional menor ou igual a 0,25, justificando a maior exclusão desses indivíduos no mercado de trabalho (Tabela 2). Estudo com pacientes com visão monocular, verificou que $44,2 \%$ dos indivíduos com indicação cirúrgica de catarata apresentavam AV menor que 0,20 e 37,7\%, AV entre 0,40 e 0,20 (15). No grupo 2, observou-se que, apesar de $52,7 \%$ dos entrevistados apresentarem AV menor ou igual a 0,25 no olho a ser operado, 79,1\% possuíam AV do olho contralateral maior que 0,25 (Tabela 2).

Assim, pressupõe-se, principalmente com pessoas com visão monocular, que os aspectos educativos relacionados à deficiência visual pela catarata e as possibilidades de reabilitação visual por meio da cirurgia, devam ser melhor trabalhados, a fim estimular a aceitação do procedimento cirúrgico por parte do paciente e de antecipar a cirurgia corretiva para evitar a exclusão social e profissional destas pessoas devido à limitação visual.

Sabe-se também da existência de barreiras socioeconômicas, psicológicas e culturais em relação à busca de assistência oftalmológica ${ }^{(4-6,16-17)}$. A aceitação do procedimento cirúrgico pelo paciente com visão monocular pode se constituir em empeciIho desde que, por já apresentar um olho funcionalmente comprometido, busca adiar a realização da cirurgia ${ }^{(17)}$.

Também é possível que para os indivíduos com catarata no único olho funcional, exista a tendência dos oftalmologistas adiarem a indicação da cirurgia, até que a visão esteja muito baixa, provavelmente, devido ao fato de existir conotação de maior responsabilidade do cirurgião nesses $\operatorname{casos}^{(18)}$. Estudo retrospectivo realizado com pacientes com tais características observou a ocorrência do dobro de complicações per-operatórias em indivíduos com visão monocular ${ }^{(15)}$. Ademais, as complicações pós-operatórias também são mais graves nesse grupo de pacientes ${ }^{(19)}$.

Estudo apontou a importância da comunicação pré-operatória com o indivíduo com visão monocular, para que as possibilidades de riscos e benefícios sejam estabelecidas e claras. Esses autores referiram tendência de indicar a cirurgia desses pacientes de forma semelhante à dos indivíduos com visão binocular, devido à importância de tratá-los em tempo hábil, não postergando a cirurgia, de forma a evitar a incapacidade de realizar atividades cotidianas ${ }^{(20)}$.

Assim, torna-se necessária, a rediscussão das indicações da cirurgia, reconhecendo e tratando também os casos em estágios iniciais, quando já apresentam algum grau de comprometimento da qualidade de vida do indivíduo. Sabe-se que há poucas décadas, indicava-se cirurgia de catarata quando a AV era de, aproximadamente, 0,10, quando a incapacidade visual já havia prejudicado muito o indivíduo. Nos últimos anos, devido aos avanços e aos bons resultados obtidos com o tratamento, a cirurgia tem sido indicada a pessoas com AV de 0,50 ou mais, a depender do comprometimento da sua qualidade de vida(21-22)

Estudo apontou como limite superior da AV, a partir da qual se indicaria a cirurgia de catarata no Reino Unido, como de 0,50, uma vez que o Sistema de Saúde do país poderia absorver tal demanda (22). Também nos Estados Unidos, vários trabalhos postulam que a AV de 0,50 é um limite adequado para a indicação da cirurgia de catarata, pois abaixo desse índice a pessoa já apresenta algumas restrições, como não estar apta a renovar a carteira de motorista ${ }^{(21)}$.

No Brasil, a literatura é escassa em relação ao limite da AV para indicação da cirurgia de catarata. Observou-se somente nos dados obtidos no grupo 2 desse estudo, coerência com os dados citados previamente, tendendo-se a indicar a cirurgia aos pacientes com AV em torno de 0,50.

No grupo 1,64,6\% dos pacientes haviam perdido a visão do olho considerado cego há mais de 5 anos. Quanto à causa da perda visual, $11,5 \%$ mencionaram não saber o motivo; $88,5 \%$ dos entrevistados tinham explicação para a baixa $A V$, sendo que 48,9\% referiram doenças específicas, como glaucoma, doença na retina ou catarata, em proporção semelhante ao que foi diagnosticado durante o exame pré-operatório, sugerindo que a maioria da amostra estudada teve acesso aos oftalmologistas e compreenderam as informações a respeito de sua condição visual, sugerindo, assim, que a postergação da cirurgia de catarata no olho funcional foi devido ou à decisão médica ou ao desejo do paciente, e não à dificuldade de acesso ao tratamento (Tabela 3).

Os entrevistados de ambos os grupos consideram insuficiente suas AVs, e, proporções significativas, afirmaram saber a causa da baixa visão no olho a ser operado (Tabela 4). Contudo, parcelas razoáveis de ambos os grupos relataram não saber a causa da "visão fraca", sugerindo carência de esclarecimentos e/ou informações desses indivíduos. Considerando-se que no hospital estudado, no momento da indicação da cirurgia, as equipes médicas e de enfermagem esclarecem ao paciente e acompanhante acerca do problema ocular e conduta a ser tomada ${ }^{(18)}$, possivelmente, os pacientes não entenderam a explicação, talvez devido à baixa escolaridade. Entre aqueles que afirmaram saber a causa da baixa AV do olho a ser operado, observou-se que 15,9\% dos entrevistados do grupo 1 e 16,7\%, do grupo 2 não apontaram a catarata como causadora da perda visual. Provavelmente esses indivíduos também não compreenderam as informações oferecidas pela equipe de saúde no momento da indicação cirúrgica.

\section{CONCLUSÕES}

Diante dessa realidade, percebe-se que crenças e conhecimentos sobre a doença e suas consequências, bem como a gravidade do problema oftalmológico são fatores que interferem na busca da assistência. Ao se considerar que a catarata é responsável pelo maior número de cegos no mundo e, ao mesmo tempo, é uma doença passível de recuperação por tratamento cirúrgico, percebe-se que é uma questão importante de saúde pública. Entretanto, nota-se que a dimensão da magnitude do problema vai além do que os oftalmologistas 
podem atingir, sendo necessário, portanto, um trabalho de cunho interdisciplinar que atinja principalmente a educação em saúde, especialmente em pessoas com visão monocular.

\section{REFERÊNCIAS}

1. Thylefors B, Négrel AD, Pararajasegaram R, Dadzie KY. Global data on blindness, Bull World Health Organ. 1995;73(1):115-21

2. Kara-Júnior N, Arieta CEL. Catarata senil. In: Kara José N, Almeida GV. Senilidade ocular. São Paulo: Roca; 2001. p. 99-107.

3. Delgado AMN, Kara-José N. Projetos comunitários em Oftalmologia. In: Kara José N, Delgado AMN, Arieta CEL, Rodrigues MLV, Alves MR. Prevenção da cegueira por catarata. Campinas: Editora da Unicamp; 1996. p.55-70.

4. Kara-José N, Arieta CEL, Temporini ER, Kang KM, Ambrósio LE. Tratamento cirúrgico da catarata senil: óbices para o paciente. Arq Bras Oftalmol. 1996;59(6):573-7.

5. Kara-Júnior N, Schellini SA, Silva MRBM, Bruni LF, Almeida AGC. Projeto catarata qual a sua importância para a comunidade? Arq Bras Oftalmol. 1996:59(5):490-6.

6. Temporini ER, Kara N Jr, Kara-José N, Holzchuh N. Popular beliefs regarding the treatment of senile cataract. Rev Saúde Pública. 2002;36(3):343-8.

7. International Council of Ophthalmology. Visual Standards: aspects and ranges of vision loss with emphasis on population surveys. [Internet]. In: $29^{\text {th }}$ International Congress of Ophtalmology; 2002 Apr. Sydney, Austrália.[cited 2009 Jul 27]. Available from: http://www.icoph.org/downloads/visualstandar dsreport.pdf

8. Piovesan A, Temporini ER. Pesquisa exploratória: procedimento metodológico para o estudo de fatores humanos no campo da saúde pública. Rev Saúde Pública. 1995:29(4):318-25.

9. Temporini ER, Kara-José N. A perda da visão: estratégias de prevenção. Arq Bras Oftalmol. 2004;67(4):597-601.

10. Temporini ER, Kara-José N, Kara-Júnior N. Catarata senil: características e percepções de pacientes atendidos em projeto comunitário de reabilitação visual. Arq Bras Oftalmol. 1997;60(1):79-83.
11. Steinberg EP, Tielsh JM, Schein OD, Javitt JC, Sharkey P, Cassard SD, et al. National study of cataract surgery outcomes. Variation in 4-month postoperative outcomes as reflected in multiple outcome measures. Ophthalmology. 1994;101 (6):1131-41; discussion 1140-1. Comment in: Ophthalmology. 1994; 101 (6):977-8.

12. Campino ACC, Cyrillo DC. Situação de ocupação e renda. In: Lebrão ML. SABE - Saúde Bem-estar e envelhecimento - O Projeto Sabe no município de São Paulo: uma abordagem inicial. Brasília: Organização Pan-Americana de Saúde; 2003. p.239-55.

13. West S, Sommer A. Prevention of blindness and priorities for the future. Bull World Health Organ. 2001;79(3):244-8. Comment in: Bull World Health Organ. 2002; 80(5):419.

14. Marback RF, Temporini ER, Maia Jr OO, Schaefer T, Kara-José Jr N. Atividade ocupacional e catarata senil - opinião de pacientes de hospital universitário. Medicina, (Ribeirão Preto). 2005;38(3/4):301-9.

5. Centurion V, Carrari MJB, Caballero JC, Lavaca AC. Cirurgia da catarata em portadores de olho único. Rev Bras Oftalmol. 2000;59(8):572-6.

16. Kara-José N, Temporini ER. Cirurgia de catarata: o porquê dos excluídos. Rev Panam Salud Publica. 1999;6(4):242-8

17. Marback RF. Cirurgia de catarata: opiniões, expectativas e reações emocionais de pacientes com visão mono versus binocular [tese]. São Paulo: Faculdade de Medicina, Universidade de São Paulo; 2007

18. Kara-Júnior N. Cirurgia de catarata: aspectos clínicos e socioeconômicos [tese]. São Paulo: Faculdade de Medicina, Universidade de São Paulo; 2003

19. Trotter WL, Miller KM. Outcomes of cataract extraction in functionally monocular patients. Case-control study. J Cataract Refract Surg. 2002;28(8):1348-54.

20. Bergwerk KL, Miller KM. Outcomes of cataract surgery in monocular patients. J Cataract Refract Surg. 2000;26(11):1631-7.

21. Klein BE, Klein R, Moss SE. Incident cataract surgery: the Beaver Dam Eye Study. Ophthalmology. 1997;104(4):573-80.

22. Minassian DC, Reidy A, Desai P, Farrow S, Vadifis F, Minassian A. The deficit in cataract surgery in England and Wales and the escalating problem of visual impairment: epidemiological modeling of the population dynamics of cataract. Br J Ophthalmol. 2000;84(1):4-8. Comment in: Br J Ophthalmol. 2000;84(1):1-2.

\section{+1. \\ 36 Congresso Brasileiro de Retina e Vítreo}

\section{0 a 22 de abril de 2011 Campo Grande - MS}

Informações:

Tel.: (11) 3849-0379

E-mail: info@meetingeventos.com.br

Site: www.retina2011.com.br 